\title{
On the role of integrated computer modelling in fusion technology
}

\author{
Sergey Smolentseva ${ }^{a}$ Gandolfo Alessandro Spagnuolo ${ }^{b}$, \\ Arkady Serikov ${ }^{b}$, Jens Juul Rasmussenc, \\ Anders H. Nielsenc, Volker Naulin', Jaime Marian ${ }^{\mathrm{d}}$, Matti \\ Coleman ${ }^{\mathrm{e}, \mathrm{f}}$ and Lorenzo Malerbag,*
}

aUCLA, Mechanical \& Aerospace Eng. Dept., 48-121 E4, Los Angeles, CA 90095-1597,USA

${ }^{b} K I T$, Hermann-von-Helmholtz-Platz 1, 76344 Eggenstein-Leopoldshafen, Germany

CPPFE, Dept. Physics, Technical University of Denmark, DK-20800 Kgs. Lyngby, Denmark

dUCLA, Materials Science \& Eng. Dept., 410 Westwood Plaza, Los Angeles, CA 90095-1595, USA

eUKAEA, Culham Science Centre, Abingdon, OX14 3DB, United Kingdom

fUniversity of Cambridge, Trumpington Street, Cambridge, CB2 1PZ, United Kingdom

${ }^{g}$ CIEMAT, Energy Materials Division, Avda. Complutense 40, 28040 Madrid, Spain

lorenzo.malerba@ciemat.es

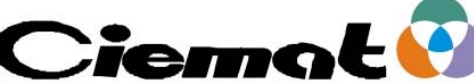

División de Materiales de Interés

Energético 


\section{Outline and Background}

- Computer modelling plays an increasingly important role in fusion design and technology:

- complexity of the physical processes involved (plasma, materials, engineering)

- highly interconnected nature of systems and components ("system of systems") call for support from sophisticated and integrated computer simulation tools.

- This presentation reviews the state-of-the-art of (coupled) computer modelling of use for reactor design (focus on breeding blanket and integrated $1^{\text {st }}$ wall) in terms of

- Neutronics

- Materials behaviour (including plasma-materials interaction, radiation effects and compatibility with fluids)

- Magnetohydrodynamics thermofluid issues and thermo-hydraulic aspects

- Simulations of plasma transport out of the confinement region to determine heat and particle loads on plasma facing components

The authors gathered together around the idea of writing such a review The presenter is only expert in one of the fields 


\section{Focus on the breeding blanket}

The blanket/FW is an in-vessel component located between vacuum vessel and plasma.

Its main purpose is threefold:

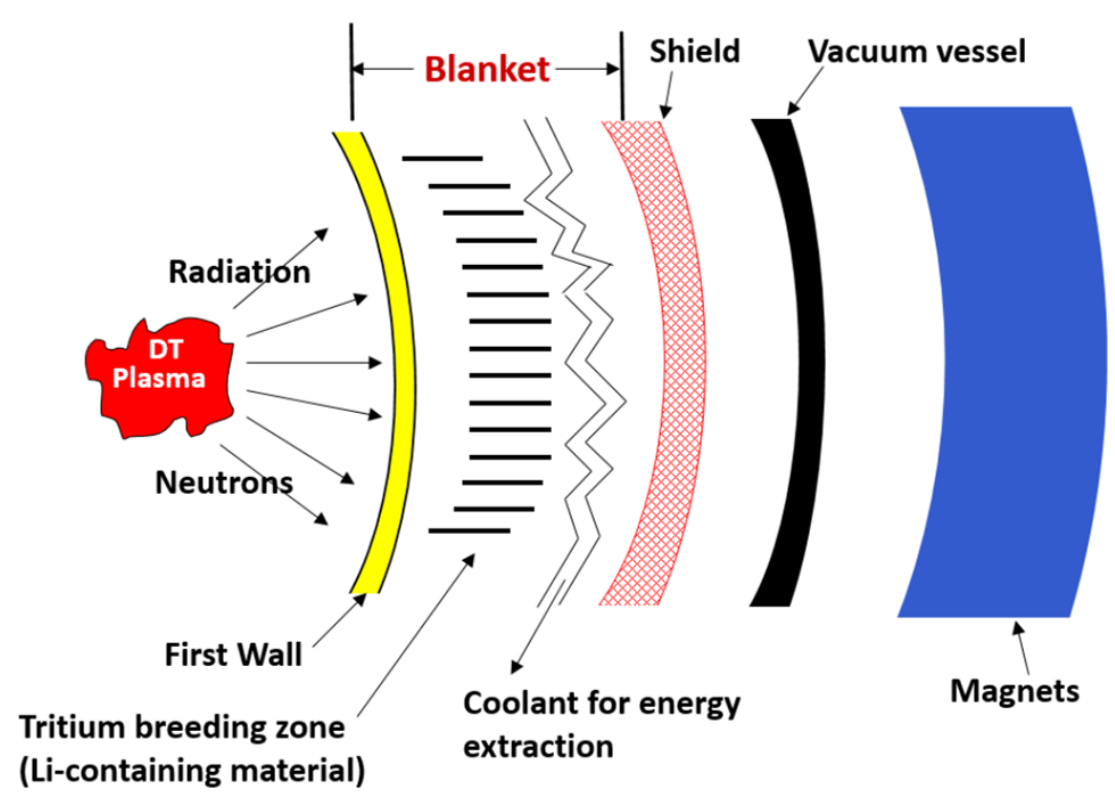

1) To assure self-sufficiency of the fusion reactor with regard to tritium (by producing, from lithium, at least the same amount of tritium as consumed in the plasma)

2) To convert fusion energy into heat (to produce electricity by using a turbinedriven generator)

3) To act as a radiation barrier (such that the components behind the breeding blanket receive the lowest possible amount of radiation)

No Breeding Blanket has ever been built or tested $\rightarrow$ crucial role of computer modelling! 
- Breeder: $L M(\mathrm{Li}, \mathrm{PbLi}), S B\left(\mathrm{Li}_{2} \mathrm{O}, \mathrm{LiAlO}_{2}, \mathrm{Li}_{2} \mathrm{ZrO}_{3}, \mathrm{Li}_{4} \mathrm{SiO}_{4}\right), M S$ (FLiBe, FLiNaBe)

- Cooling strategy: self-cooled, separately-cooled, dual-coolant

- Coolant: $L M, H e$, water

- Use of neutron multiplier: $B e, P b$

- Use of qualified or "new" materials

- Flow orientation (for LM or MS blankets): toroidal, poloidal, radial

- Location: Inboard, outboard

- Maintenance scheme: vertical, radial

- Configuration: "banana", module ( 2 m) 
- Self-cooled LM blanket

US self-cooled Li/Va blanket

- highest rank by BCSS

- low crack tolerance

- Separately cooled LM blanket

EU HCLL/WCLL blankets

- available structural materials and fabrication technologies

- T permeation from PbLi into He is a serious safety issue

- Dual-coolant LM blanket

- high thermal efficiency (>40\%)

- relies on still underqualified SiC FCl

- Molten salt blanket

- low MHD pressure drop

- low TBR and many chemistry issues

- Solid breeder blanket

- robust design

JA water-cooled SB blanket

- pebble bed thermomechanics, low thermal efficiency
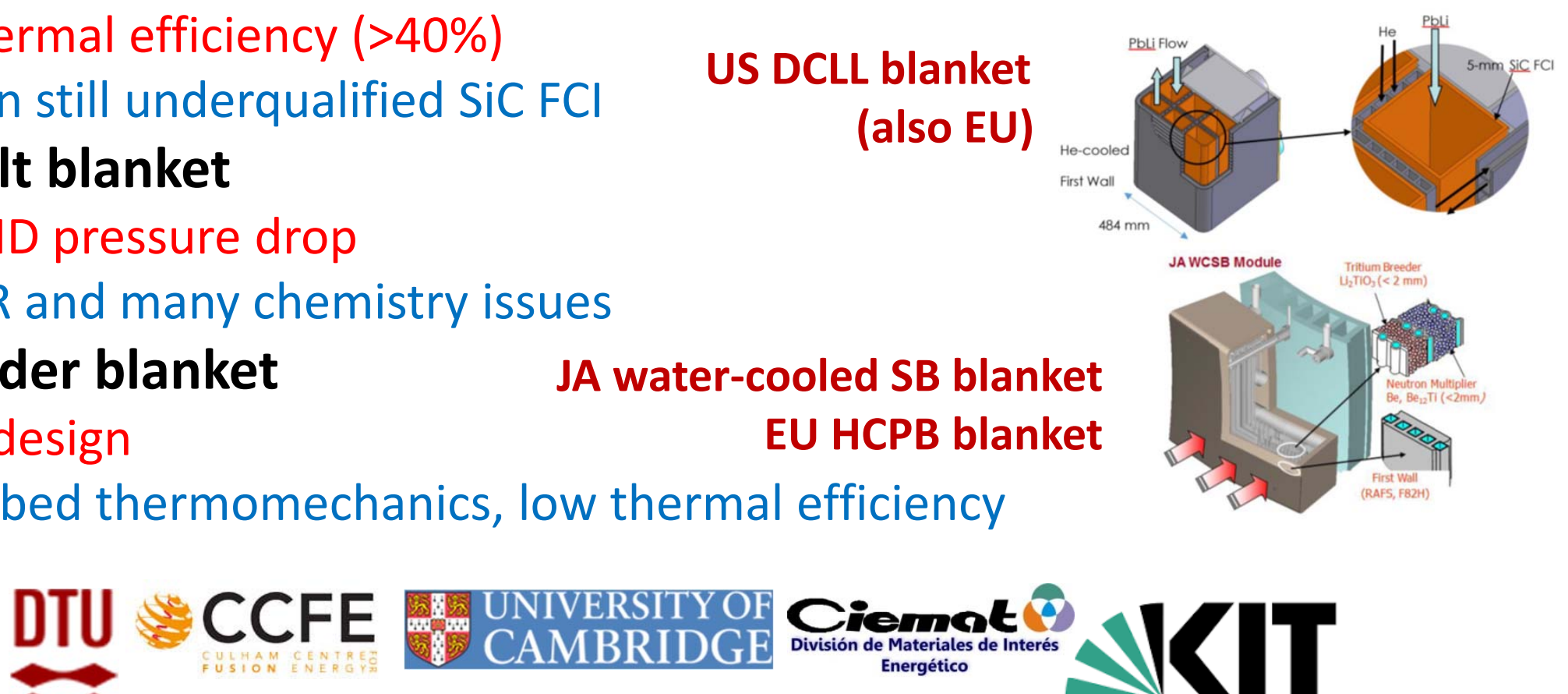


\section{Integrated modelling for fusion neutronics}

- Neutron irradiation causes activation of the structural materials up to the bioshield, with wide distribution of the radioactive sources, including activation of cooling water.

- Neutronics specifics \& challenges of large fusion tokamaks and stellarators :

- Complicated 3D geometry of substantially heterogeneous CAD models

- Large volumes of $14 \mathrm{MeV}$ neutron sources in D-T plasma

- Monte Carlo (MC) method of radiation transport is most suitable for such complex geometry, especially for geometries with channels and gaps which open radiation streaming pathways

- Use of CAD-to-MC geometry conversion is an inevitable part of the neutronics modelling process

- Decay gamma transport must be taken into account for Shut-Down Dose Rate (SDDR) calculations challenge to combine neutron transport and activation

- Two integrated modelling approaches have been developed, T1 and T2:

- Type T1 is CAD-to-MC geometry conversion modeling which allows the most accurate reproduction of the actual design and closest correspondence to reality to be achieved

- Type $\mathbf{2} 2$ is the methodology of Shut Down Dose Rate calculations including transport of decay gammas from distributed gamma sources
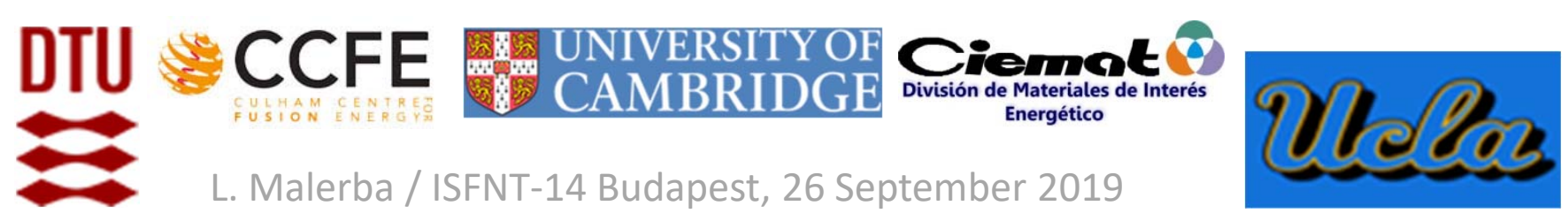


\section{T1 integrated modeling: CAD-to-MC geometry conversion illustrated on the McCad modeling approach}

Stages and processes of the CAD-to-MC model conversion with the McCad software [Ref. 1, 2] McCad is available freely on github: https://github.com/McCadKIT/FreeCAD-McCad

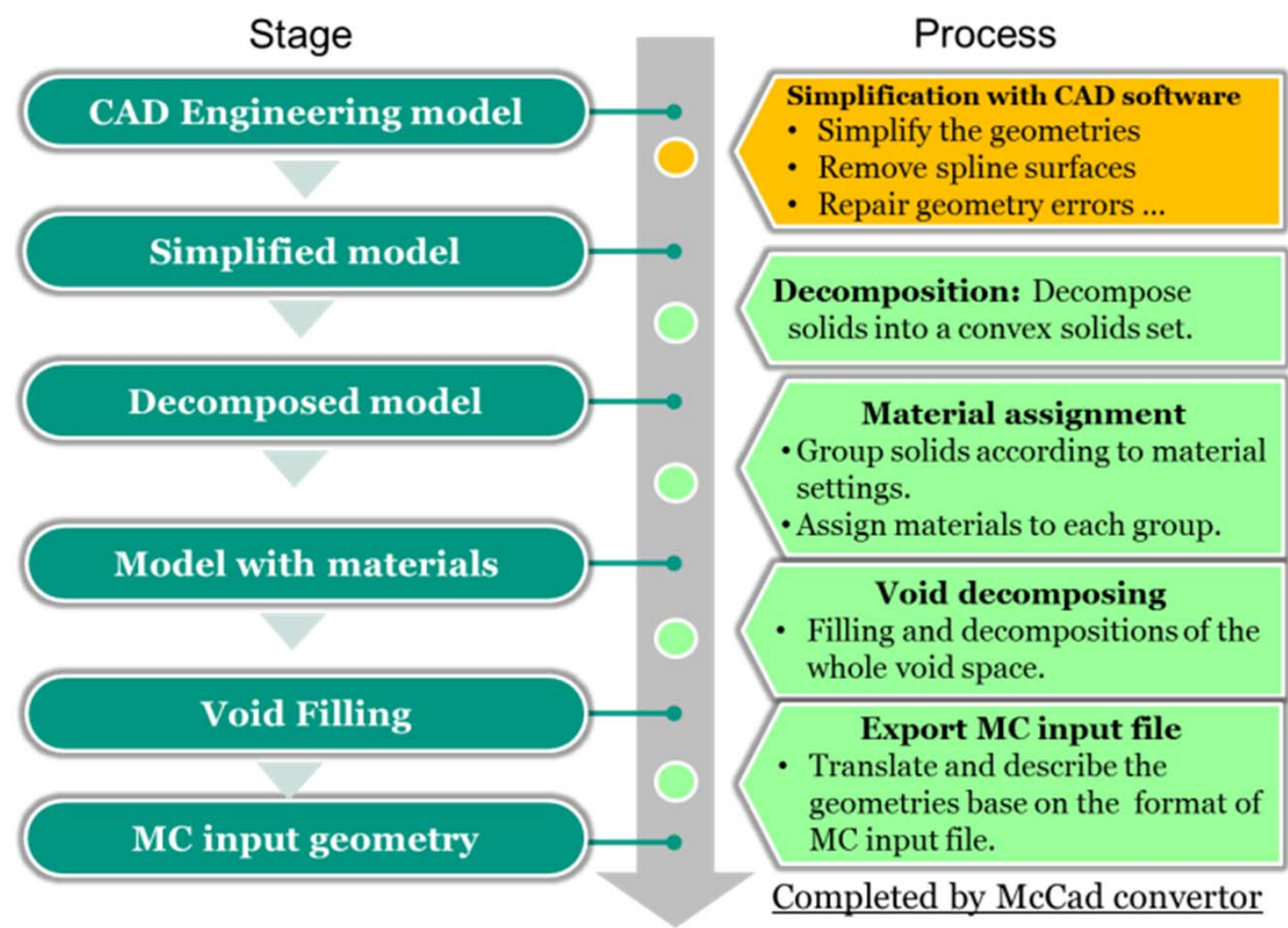

T1 using the MC model is suitable and sufficient for the following calculations:

- neutron and photon fluxes

- nuclear heating (neutron and secondary photons)

- neutron damage (dpa)

- helium and hydrogen gas production

[Ref. 1] D.Grosse et al., Status of the McCad geometry conversion tool and related visualization capabilities for 3D fusion neutronics calculations, Fusion Eng. Des. 88 (2013) 2210-2214.

[Ref. 2] L. Lu et al., Development of McCad as an Integrated Interface Tool for the CAD to MC Geometry Conversion, M\&C 2017, Jeju, Korea, April 16-20, 2017. 
The MC model produced as result of $\mathrm{T} 1$ is supplied as input of $\mathrm{T} 2$, which integrates $\mathrm{MC}$ radiation transport and activation inventory calculation of the distributed decay gamma sources, and decay gamma transport, for SDDR calculations

1. MCNP neutron transport calculations to assess the spatial distribution of neutron spectra with mesh-tallies - STEP 1

FISPACT activation calculations using mesh-tallied neutron spectra and irradiation scenario:

- to quantify the generation of the radioactive nuclides in different materials;

- formation of the 3D decay gamma source covering a facility space of interest at required shutdown time

2. MCNP decay photon transport calculation to get the response from the source in the volume of interest and corresponding Shut-Down Dose Rates (SDDR) - STEP 2
T2 has to be used for the calculation of:

- material activation

- transmutation

- detailed radioactive waste management

- Shut-Down Dose Rate

[Ref. 3] M. Majerle et al., Verification and validation of the R2Smesh approach for the calculation of high resolution shutdown dose rate distributions, Fusion Eng. Des. 87 (2012) 443-447.

[Ref. 4] P. Pereslavtsev et al., Novel approach for efficient mesh based Monte Carlo shutdown dose rate calculations, Fus. Eng. Des. 88 (2013) 2719-2722 


\section{Example of neutronics analysis with two types of integration}

\section{T1 (CAD-to-MCNP conversion) T2 (SDDR calculation with R2Smesh)}

Example for SDDR analysis of the ITER Core Imaging X-Ray Spectrometer (CIXS) (Diagnostic system installed in ITER equatorial port)

T1 is sufficient for the following calculations:

- neutron and photon fluxes

- nuclear heating (neutron and secondary photons)

- neutron damage (dpa)

- helium and hydrogen gas production

T2 should be used for the for the following tasks:

- material activation

- transmutation

- detailed radioactive waste management

- Shut-Down Dose Rate (SDDR) calculations

The detail T1 \& T2 results of the ITER CIXS neutronics analysis: [Ref. 5] A. Serikov et al., Neutronics analysis for the ITER core imaging X-ray spectrometer, Fusion Eng. Des. 109-111 (2016) 848-854

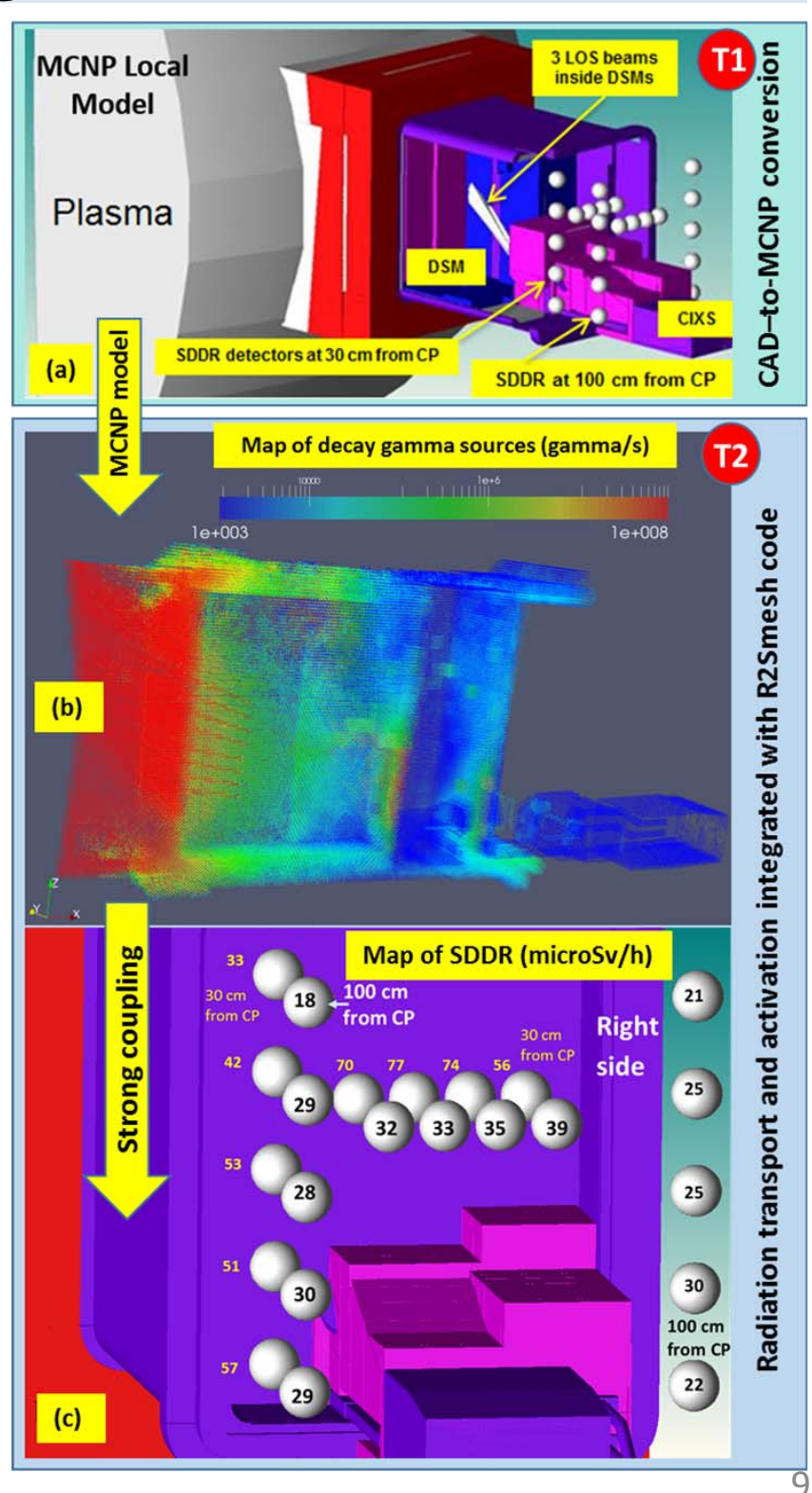




\section{Need for integration: neutronics and heat loads \& transfer}

- Conventional coupling procedure used for the BB design:

- Creation of generic CAD

- Preparation of neutronic model (geometry details are not nodalised)

- Neutronic output (i.e. radial power density) used for thermal-hydraulic and structural analysis without feedback

- Drawbacks:

- Different geometry used by different models

- The output of subsequent model does not feed back the previous one

- Different teams with different background involved in the design

- Time consuming and herald of errors.
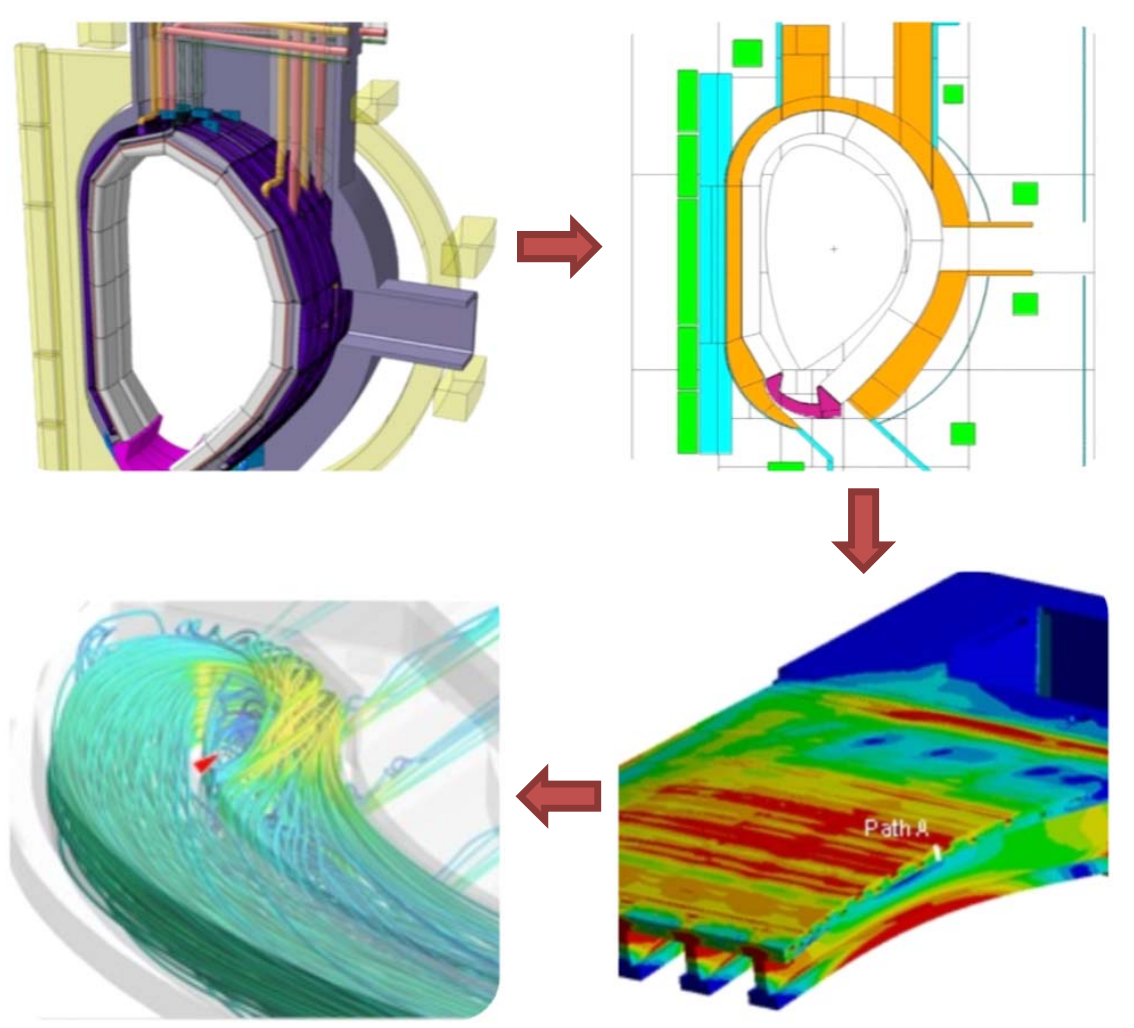


\section{MAIA = Multi-physics Approach for Integrated Analysis \\ Coupling procedure}
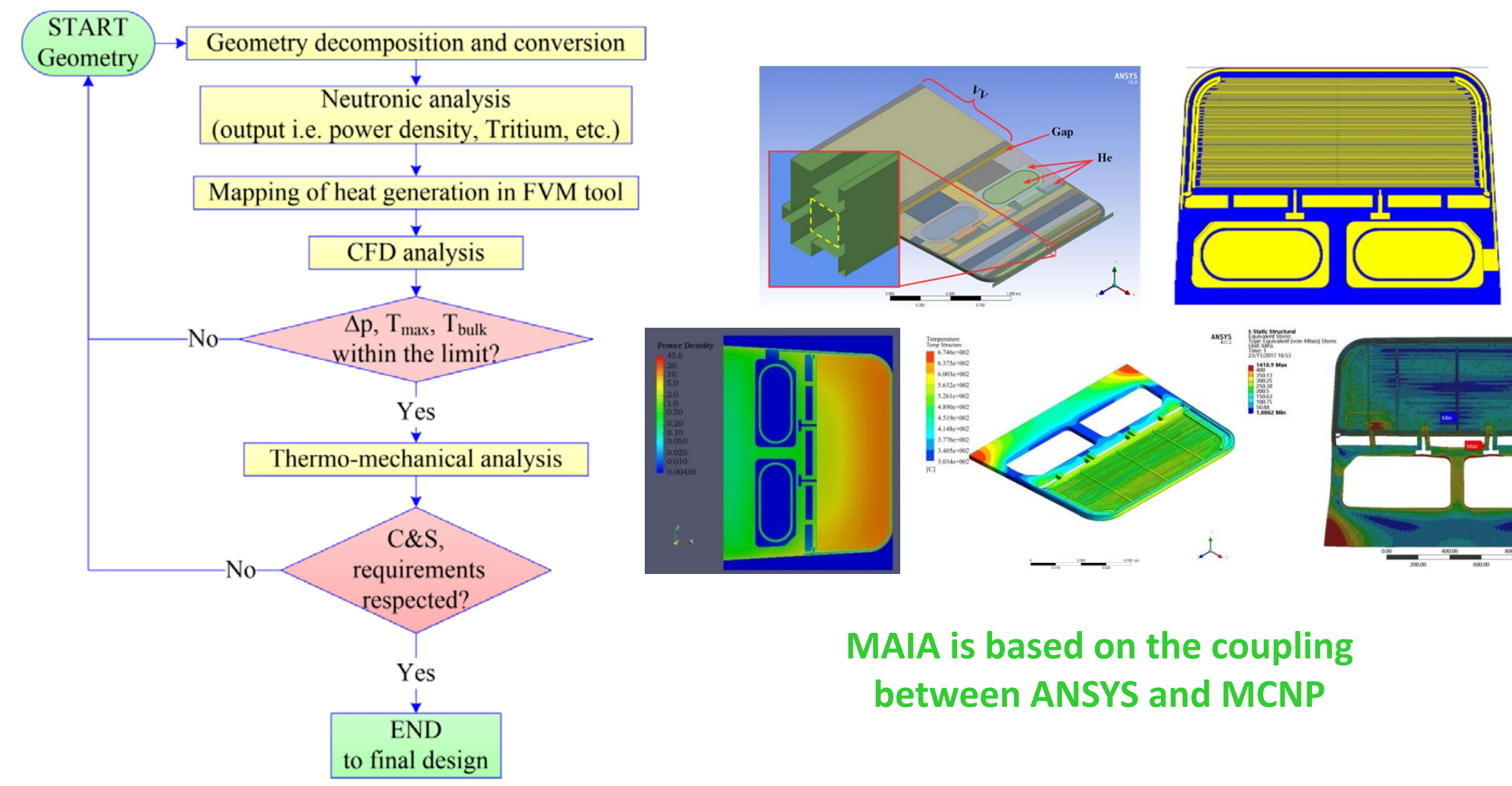

MAIA is based on the coupling between ANSYS and MCNP

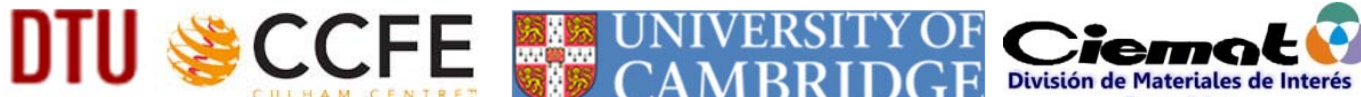




\section{Enhancement of MAIA procedure}
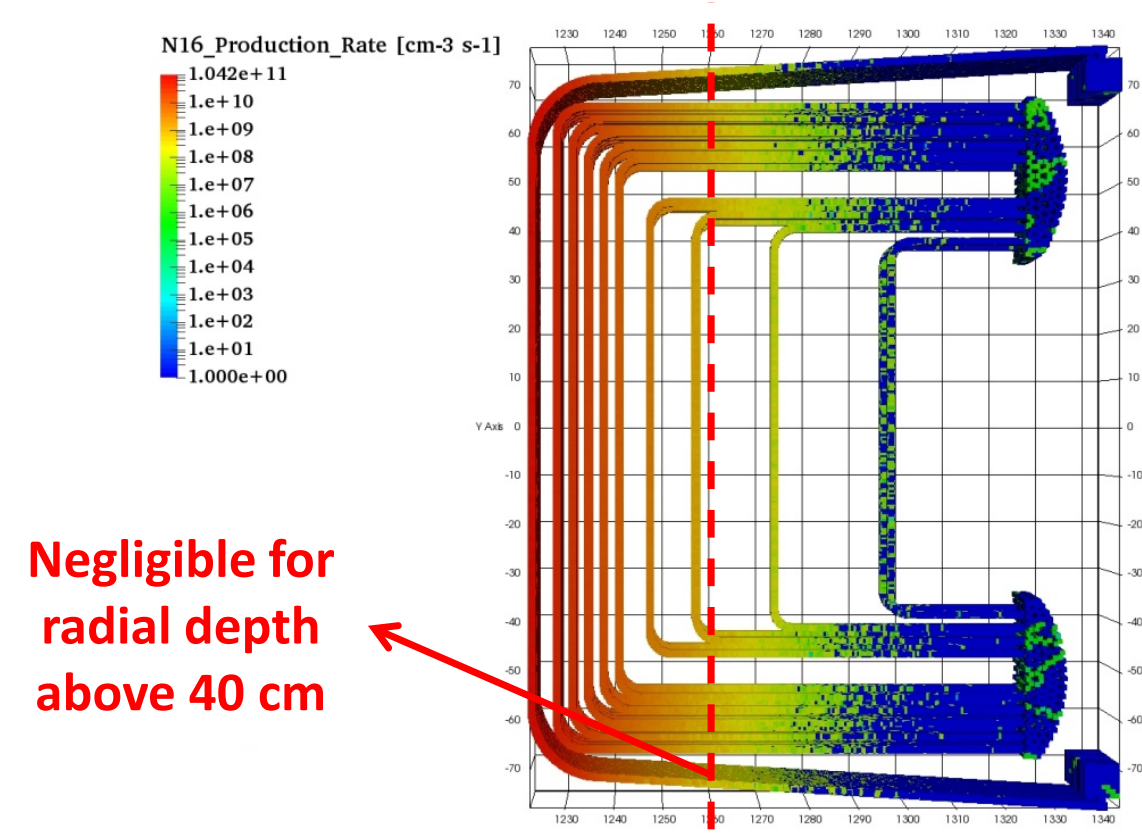

Concentration $[\mathrm{Cl}$ $1.054 e+011$
$9.894 e+010$ $9.894 \mathrm{e}+010$ $9.252 \mathrm{e}+010$ $8.609 \mathrm{e}+010$ $7.967 \mathrm{e}+010$ $7.324 \mathrm{e}+010$ $6.682 \mathrm{e}+010$ $6.040 \mathrm{e}+010$ $5.397 \mathrm{e}+010$ $4.755 e+010$ $4.112 \mathrm{e}+010$ $3.470 \mathrm{e}+010$ $2.827 e+010$ $2.185 \mathrm{e}+010$ $1.542 \mathrm{e}+010$ $8.998 \mathrm{e}+009$ $8.998 \mathrm{e}+009$ $2.574 \mathrm{e}+009$

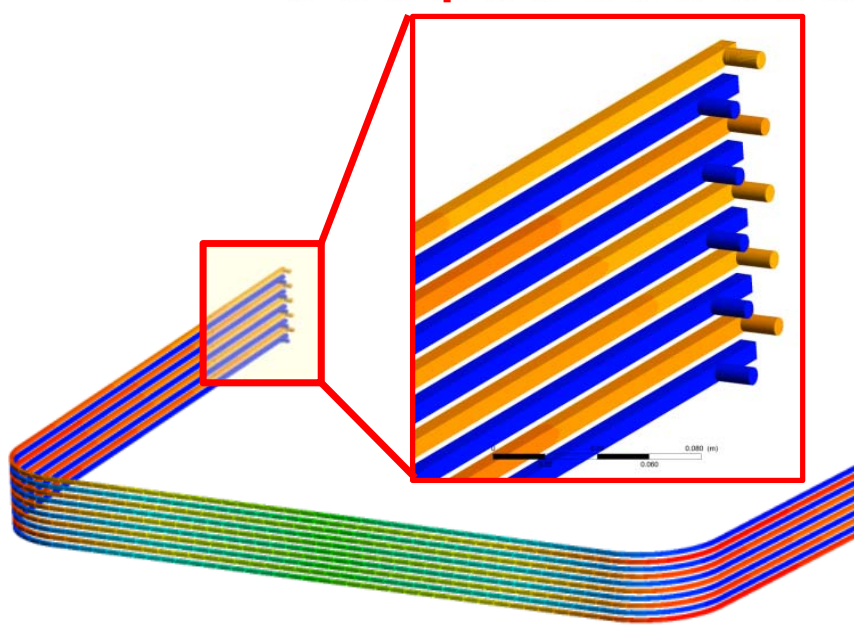

\section{Application of MAIA procedure for water activation analysis.}

Direct coupling of neutronic analysis ( $\mathrm{N}$ isotopes production rate) and CFD calculation (3D N concentration distribution).

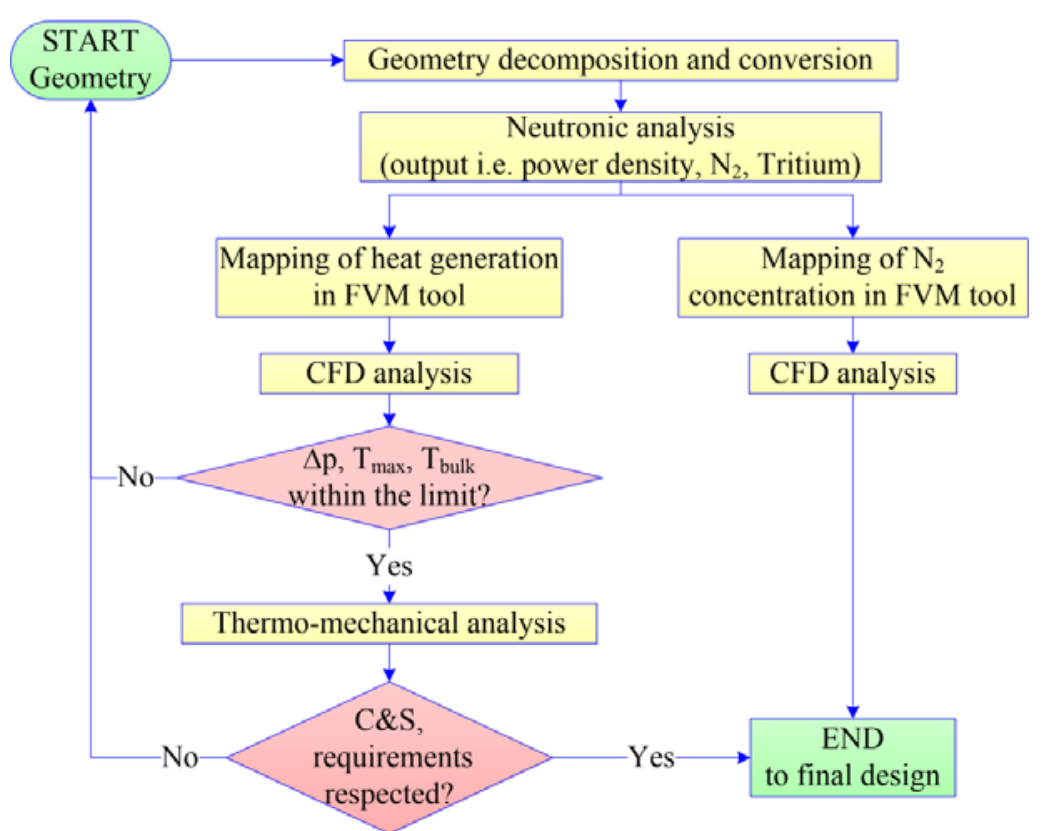

P. Chiovaro et al., Investigation of the DEMO WCLL Breeding Blanket Cooling Water Activation (POSTER)

I. Moscato et al., Assessment of the Dose Rates due to Water Activation on an Isolation Valve of the DEMO WCLL Breeding Blanket Primary Heat Transfer System (POSTER) 


\section{Neutronics open issues are more experimental than computational}

- Volumetric neutron source from D-T plasma. Right now we have $14 \mathrm{MeV}$ neutrons from point D-T sources of neutron generators, accelerator-driven systems (ADS), Hohlraum capsule of National Ignition Facility (NIF), and so on. In all devices the source size is small.

- Tritium breeding for D-T fusion facilities beyond ITER. Requirement of tritium fueling system for TBR is 1.05. Engineering requirement for TBR is higher by $10 \%$, it is 1.15 due to the need to compensate installation of non-breeding systems inside the ports with antennas of ICH, ECH systems, diagnostic systems, and so on.

- 11 modelling challenges to reproduce CAD-based geometry and all known physics phenomena are used in neutronics simulations in the form of nuclear cross-section data or models. The Monte Carlo (MC) method of radiation transport is the most suitable for complex, substantially heterogeneous geometry of fusion devices.

- 12 activation-transport coupling schemes for SDDR. The possibility to get the experimental results gained from facilities operated with D-T source is very dependent on maintenance access to particular areas, such as Inter-Space Structure (ISS) behind the port plugs. The safety of maintenance access is defined by the values of shut-down dose rates (SDDR) at those areas. Therefore, SDDR should be accurately estimated, taking account radiation transport and time-dependent activation phenomena. 


\section{Materials operating conditions}

Thermal and damage gradients $\rightarrow$ internal stresses $\rightarrow$ cracking (integrity of interfaces/welds ?)
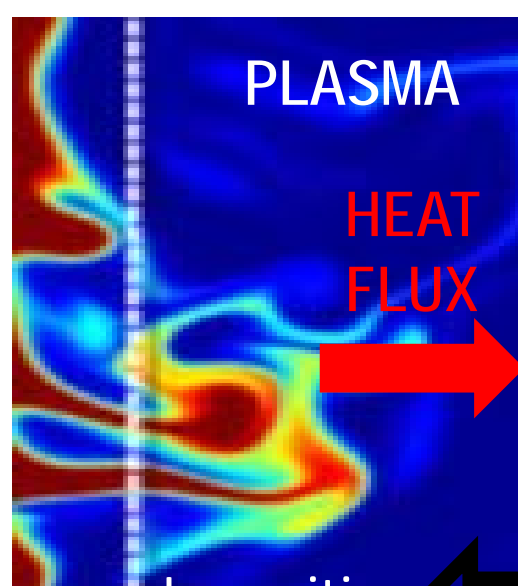

Impurities
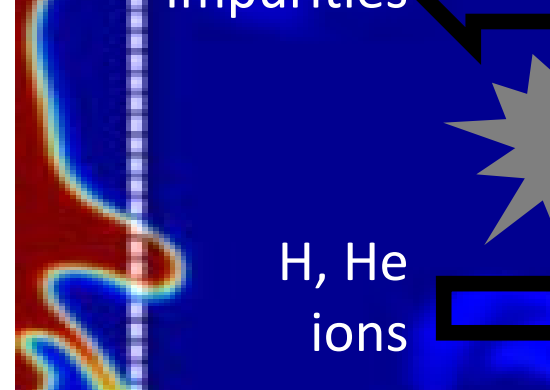

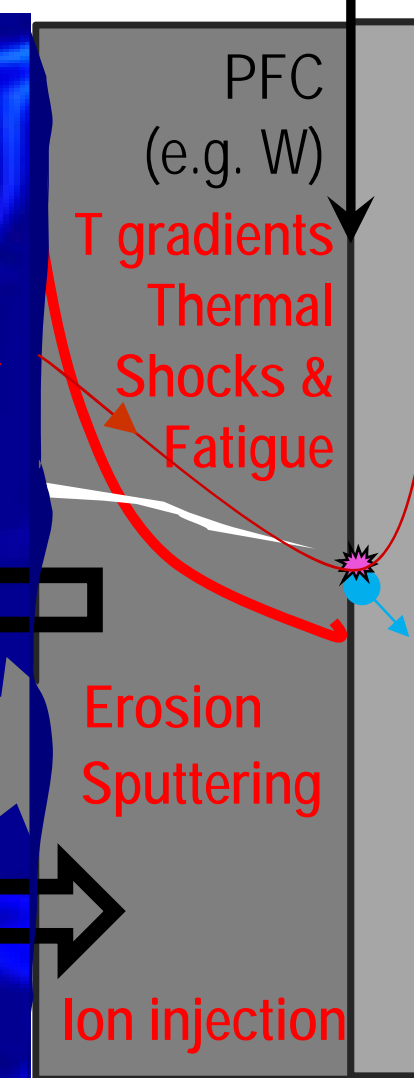

$$
\text { (e.g. Eurofer) }
$$

Neutron

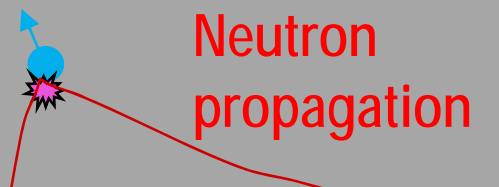

Displacement

Damage

Transmutation

(He, H)

Swelling \& irradiation creep

Hardening \&

Embrittlement

T permeation and

corrosion protection barrier (if relevant)

\section{Flowing fluid}

Water: Generalized

corrosion

Helium Effects of

impurities,

especially C, N, ...

Liquid metal:

Dissolution, LME

(are barriers

enough?) 


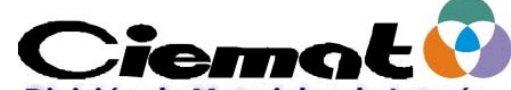

División de Materiales de Interés

Energético

\section{Radiation effects - multiscale materials \\ modelling}

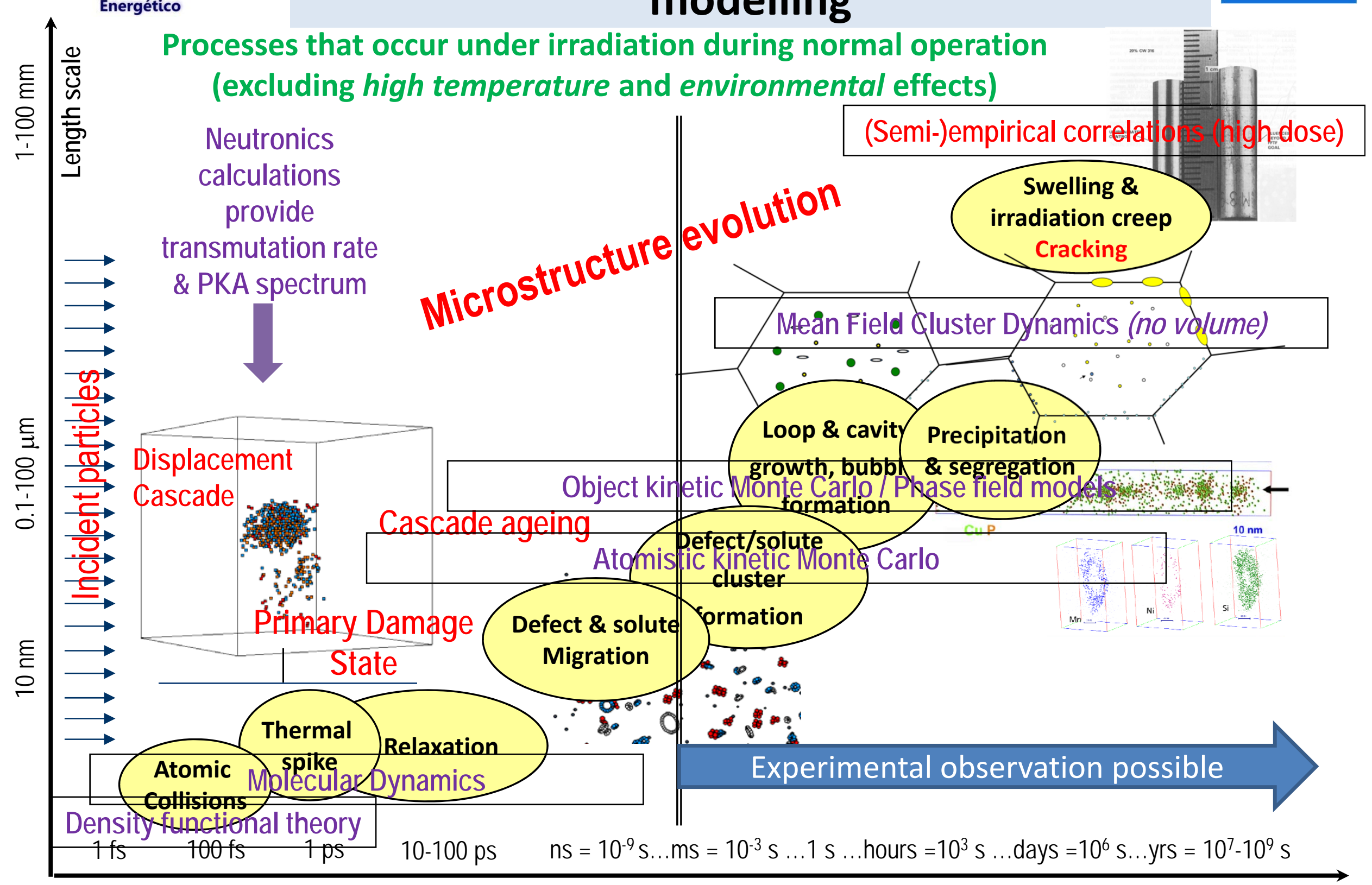




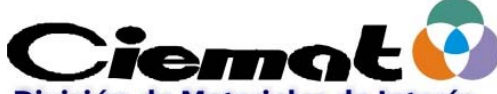

División de Materiales de Interés Energético

\section{What happens if we test a material after irradiation? Change of mechanical properties}

Timescale not really meaningful: strain at given strain-rate is the evolution variable
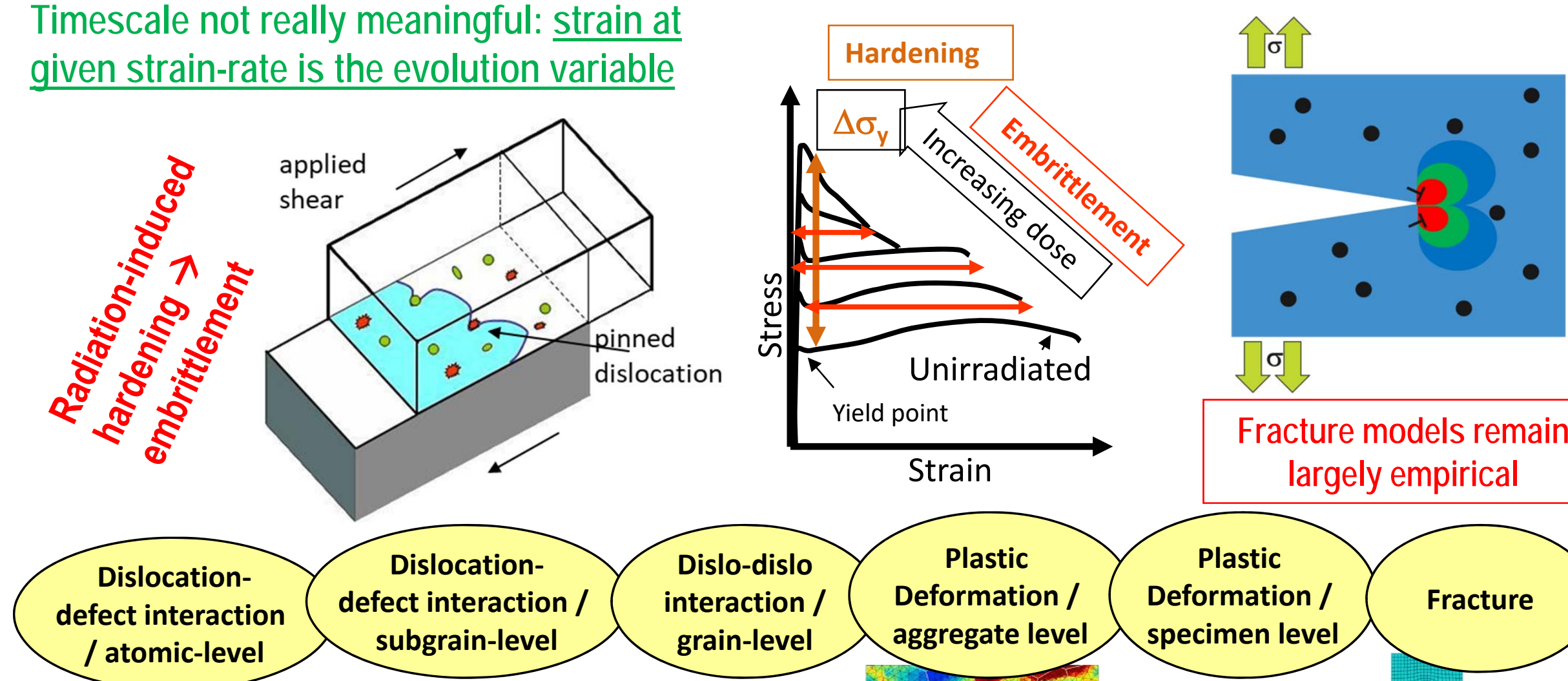
Dislo-dislo interaction / grain-level Plastic Deformation / aggregate level Increasing empirieism

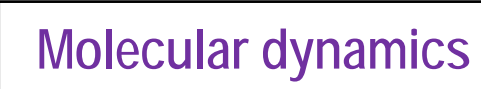

$10-100 \mathrm{~nm}$

\section{Dislocation dynamics}

1-100 $\mu \mathrm{m}$

$1-100 \mathrm{~mm}$

Fracture models remain largely empirical

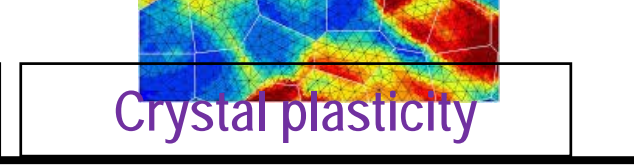

Continuum mechanics

\section{Plastic} Deformation / specimen level Fracture
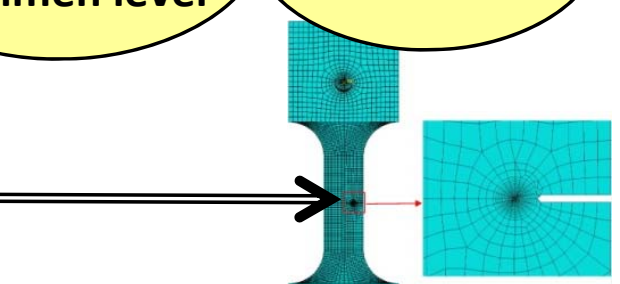

$0.1-1 \mathrm{~m}$

Length scale

(2) 


\section{Hypothetical couplings for a multiscale blanket materials modelling}

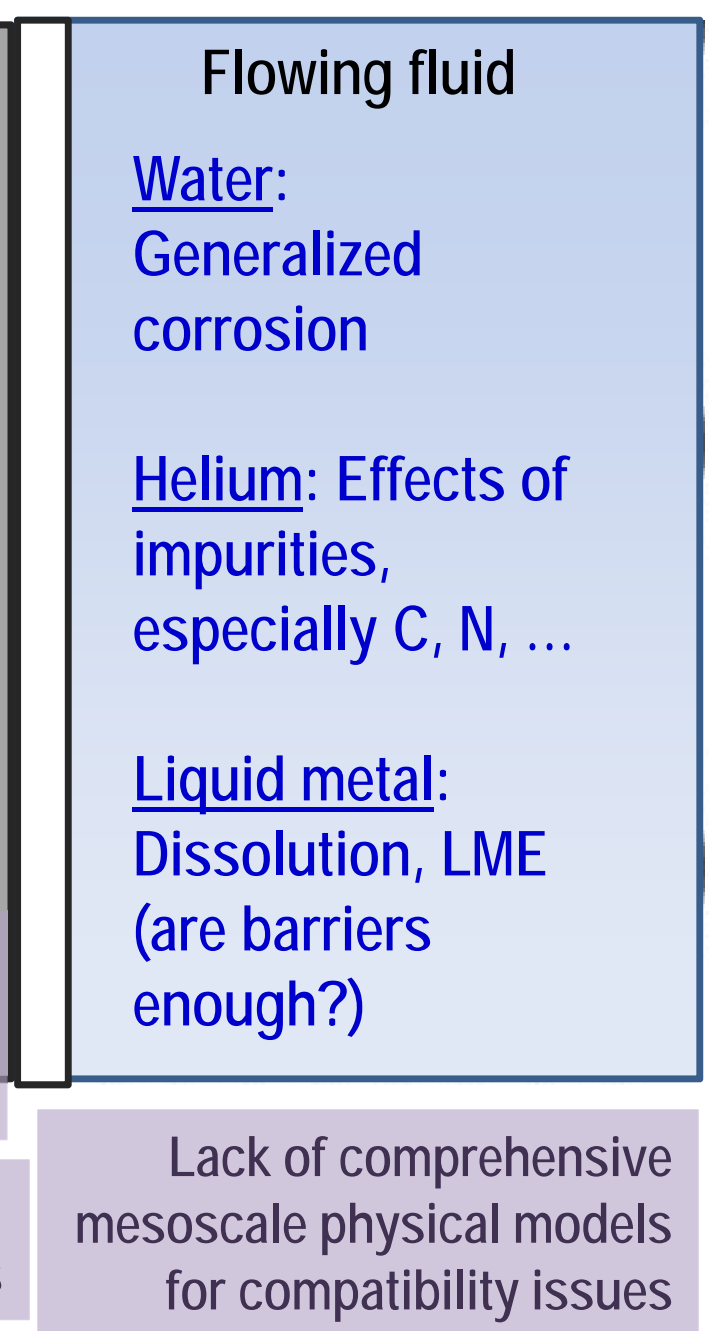




\section{Open issues and new trends}

- Connect different scales:

- Currently multiscale modelling = sequential use of single-scale tools with input from higher fidelity models

- Allow for effects of heterogeneity and chemical complexity:

- Contradiction between averaging at higher scale and local phenomena triggering e.g. crack initiation

- Lack of full understanding of physical mechanisms:

- True for most compatibility issues (e.g. IASCC, LME ... effects of localisation? See above)

- Computational cost:

- Limited representative volumes/timescales accessible to high fidelity models

- New trends:

- Machine learning and data-driven modelling

- Possible strategy:

- Filter information, remain with 1st order effects - physics cannot be forgotten

- Take local information to higher scale (with the the help of machine learning?)

- Deduce simple laws from complex problems (again with the help of machine learning?) 


\section{Liquid metal blanket design: MHD and Heat \& Mass Transfer (transport processes) are primary drivers}

$>$ MHD. The motion of electrically conducting breeder/coolant in magnetic field induces electric currents, which interact with the magnetic field, resulting in strong Lorentz forces ( 4 to 5 orders of magnitude higher than hydrodynamic forces) that modify the flow in many ways.

$>$ Heat transfer. The flowing LM and the surrounding structure absorb volumetric and surface heat resulting in high, stronggradient temperature field in the liquid and the solid.

$>$ Mass transfer. (1) Li or PbLi are chemically aggressive, causing corrosion of structural and functional materials. (2) Once generated, Tritium is conveyed by the flowing LM and diffuses through the liquid and the solid.

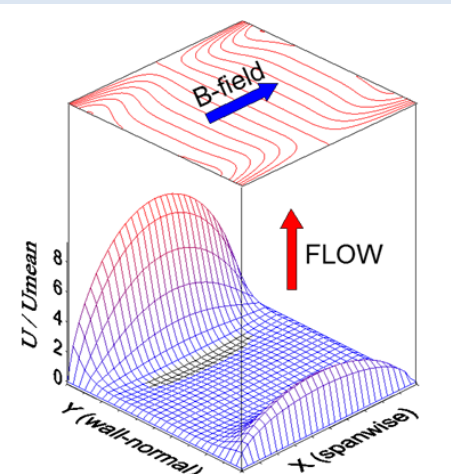

Velocity and induced currents in MHD flow in a duct
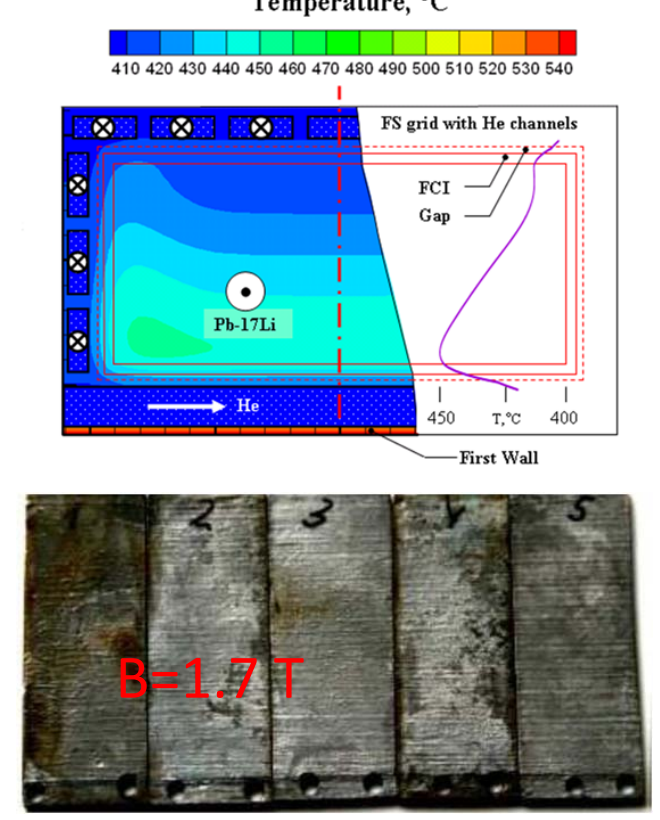

Corrosion of RAFM in PbLi under B-field field in DCLL (ITER TBM) 
In a LM blanket, MHD and Heat \& Mass Transfer are non-linearly coupled leading to multiple effects

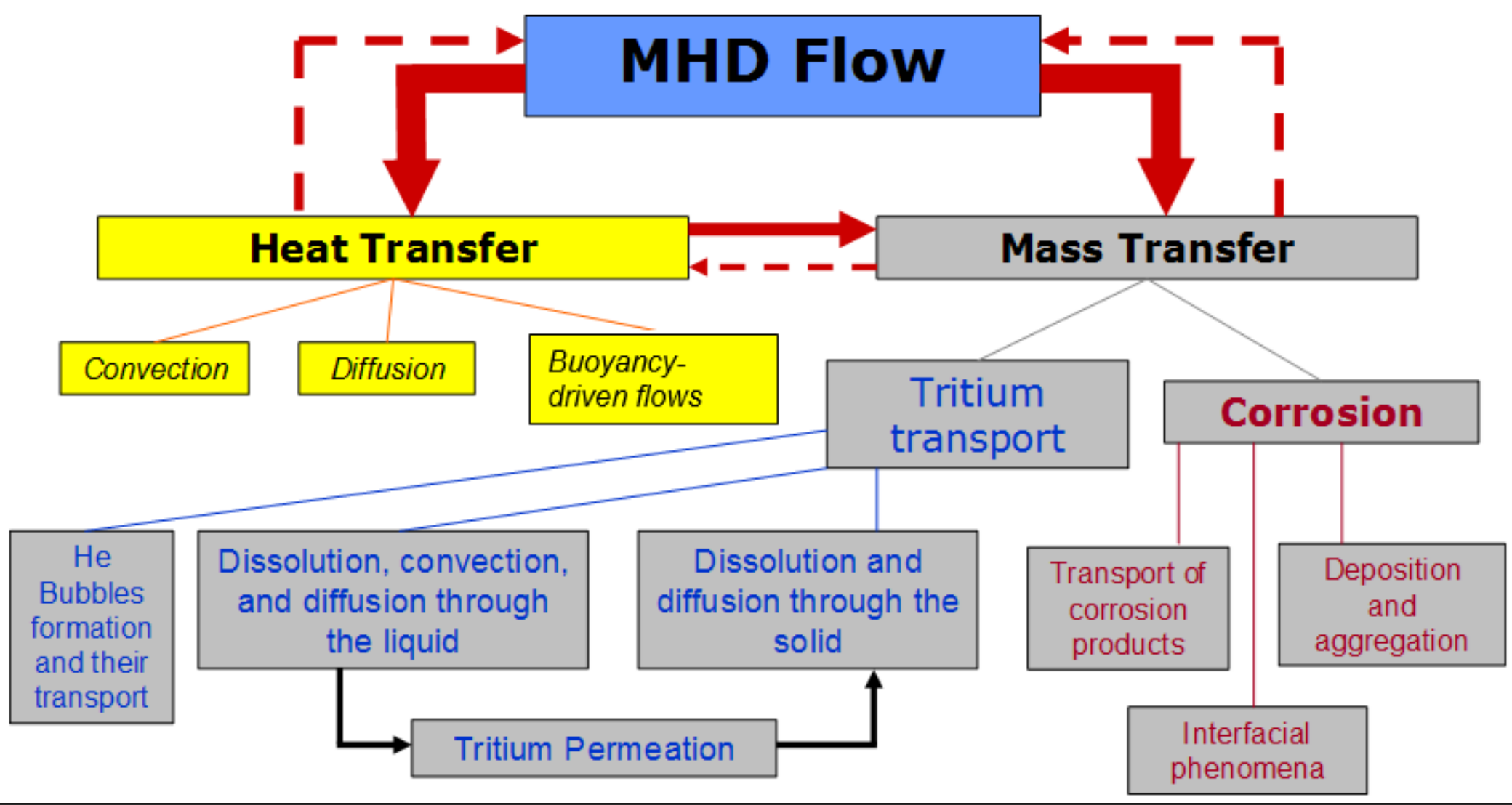

The simulation of these multiple effects in a blanket requires

INTEGRATED MODELING TOOLS! 


\section{Which Computational Magneto- HydroDynamics tools are presently available?}

\begin{tabular}{|c|c|c|}
\hline $\mid$ & $\begin{array}{l}\text { Commercial multi- } \\
\text { purpose CFD codes } \\
\text { nith buil-in/user- } \\
\text { defined MHD } \\
\text { module }\end{array}$ & $\begin{array}{l}\text { FLUENT - commercial multi-purpose CFD solver with a built-in } \\
\text { MHD module } \\
\text { CFX - commercial multi-purpose CFD solver with a user-developed } \\
\text { MHD module } \\
\text { SC/TETRA - commercial multi-purpose CFD solver with a built-in } \\
\text { MHD module } \\
\text { OpenFoam - open-source multi-purpose CFD solver with a build-in } \\
\text { electrodynamics module or user-developed MHD module }\end{array}$ \\
\hline & $\begin{array}{l}\text { Massive non- } \\
\text { commercial "home- } \\
\text { madle" solvers, } \\
\text { especially developed } \\
\text { for M-D applications }\end{array}$ & $\begin{array}{l}\text { FLUIDYN - CFD and multi-physics solver with build-in MHD } \\
\text { capabilities by TRANSOFT International } \\
\text { UCAS (China) - "home-made" MHD solver with many } \\
\text { computational capabilities } \\
\text { HIMAG (USA) - "home-made" MHD solver with many } \\
\text { Computational capabilities } \\
\text { FEMPAR (Spain, Badia et al.) - "home-made" multi-physics solver } \\
\text { with MHD capabilities }\end{array}$ \\
\hline & $\begin{array}{l}\text { Research codes } \\
\text { limited to special type } \\
\text { of flow and/or simple } \\
\text { flow geometries }\end{array}$ & $\begin{array}{l}\text { rch codes, e.g. CoreFlow (L. Bühler, } \\
\text { (S. Smolentsev, US) } \\
\text { ake/Kunugi (Japan), Krasnov/Zikanov/Bueck }\end{array}$ \\
\hline
\end{tabular}




\section{Where are we now in CMHD?}
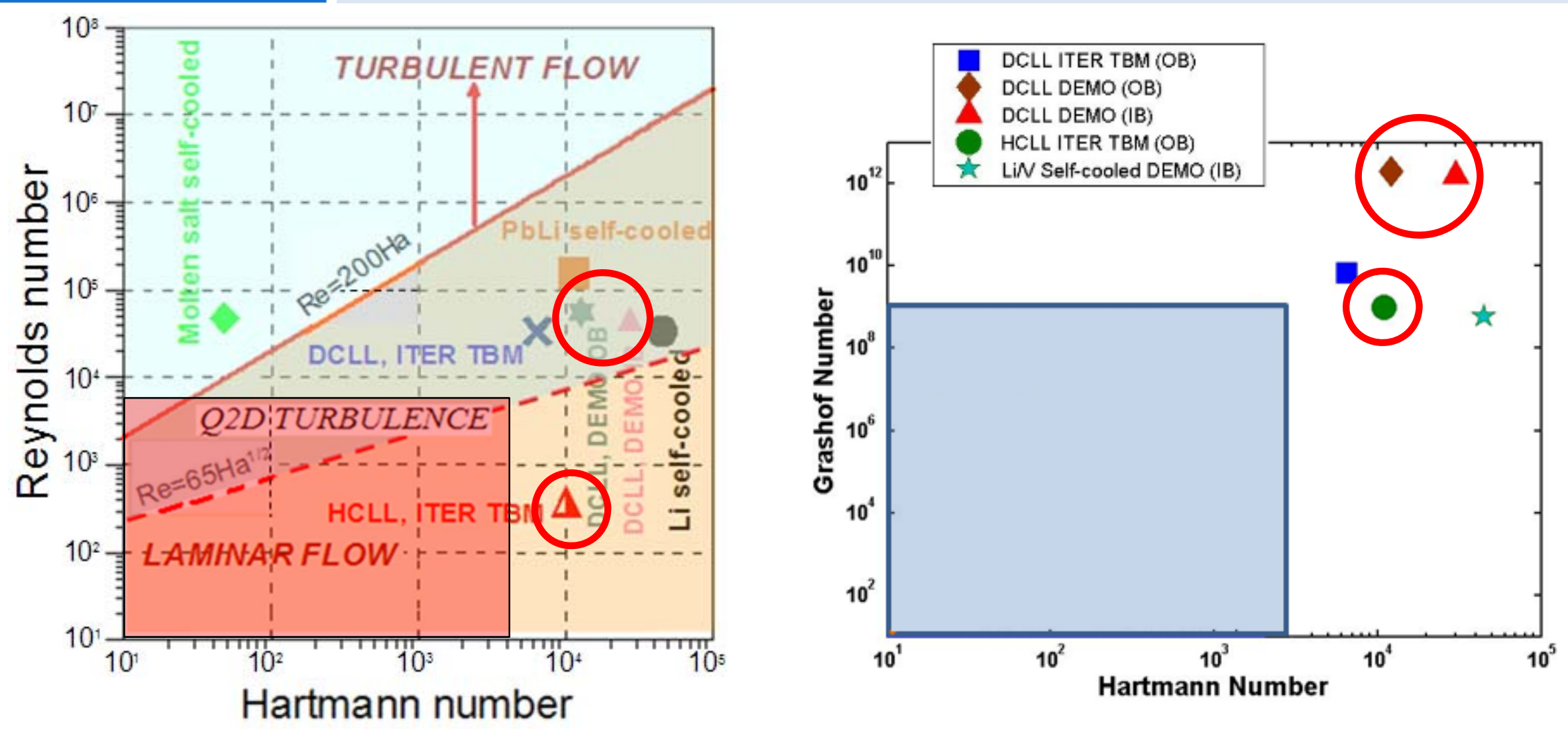

We are still pretty far from the target numbers 


\section{DTU \\ On the other side of the blanket/divertor: heat transfer from the plasma}

Courtesy EFDA/JET

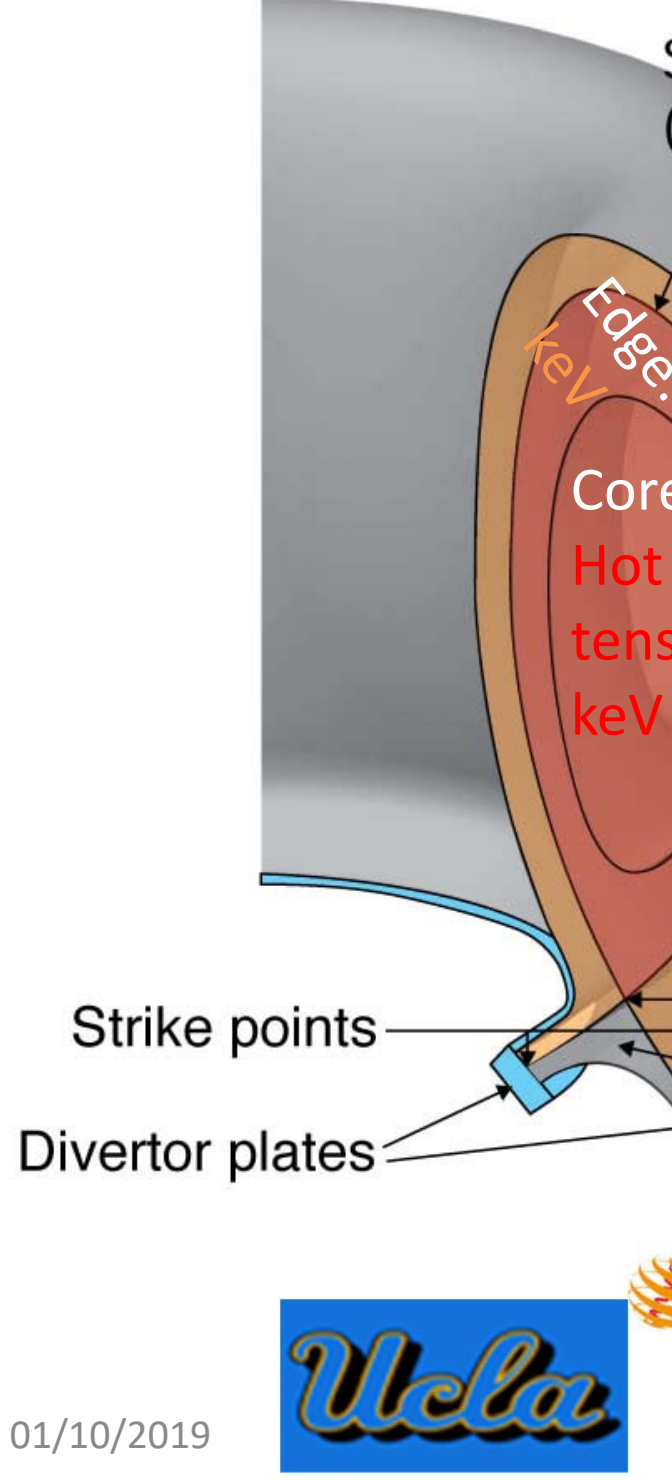

Separatrix

(i.e LCFS)

SOL:

$>$ Exhaust

Fuelling

$>$ Recycling

Closed magnetic surfaces

Main transport channel from confined plasma to Open SOL out-board-midplane magnetic surfaces

Scrape-off layer, SOL Cold: tens of eV 


\section{DTU SOL transport is non-diffusive - Strongly intermittent}

Cross field transport of particles and heat in magnetically confined hot plasmas is dominated by anomalous - turbulent - transport!

In the edge/SOL region the transport is strongly intermittent and characterized by:

$>$ Large-amplitude, radially propagating magnetic field aligned filamentary structures - blobs - of elevated plasma pressure generated close to the last closed flux surface

$>$ Resulting in localized power loads at plasma facing components

$>$ Lasting influence on the chamber wall and other plasma facing components - particularly the divertor structures

$>$ Strong demands on materials

Observed under a variety of conditions:

Zweben Phys. Fuids 28974 (1985); Zweben et al. PPCF 49, S1 (2007); Garcia, Plasma Fusion Res. 4, 019 (2009); D'Ippolito et al. Phys Plasmas 18, 060501 (2011); Vianello et al. Nucl. Fus. 57116014 (2017).

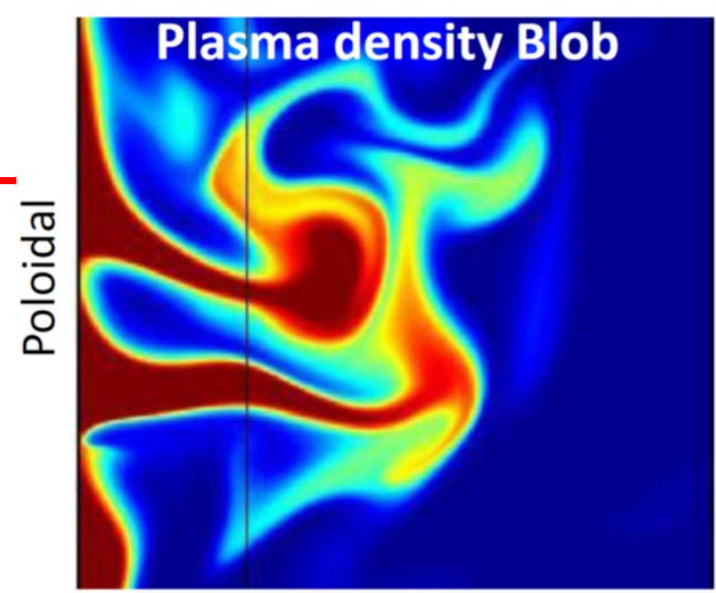

Radial
CCFE HNIVERSITY OF Ciermere

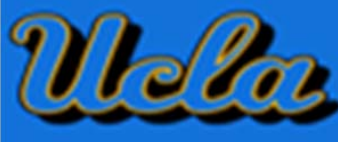




\section{DTU Modelling the SOL transport and plasma exhaust - wishlist}

$>$ Multiscale - from the parallel connection length (tens of meters) over the SOL plasma gap $(5-10 \mathrm{~cm})$, down to the power fall off length into the SOL and the wall sheath width $(\mathrm{mm}$ ) and the ion Larmor radius (sub $\mathrm{mm}$ )

> Multiphysics - population of "active" neutrals needs account for plasma / neutral interactions- elastic as well as in-elastic collisions - ionization, excitations etc.

$>$ Multi-ion species -various hydrogen isotopes, helium, ions from gas puffs, impurities from sputtering of PFC.

$>$ Models must encompass the edge and SOL region across Last Closed Flux Surface

$>$ No separation between fluctuations and profiles - evolved on the same footing - fully non-linear description.

$>$ SOL - open field lines end on material surfaces demanding sheath boundary conditions - may be implemented in a fluid description; but issues as detachment demand kinetic descriptions. 


\section{Edge/SOL dynamical codes}

Several codes have been developed and applied during the last couple of decades

Typically based on four-field, drift-fluid models for vorticity, density, and electron and ion pressure with

Braginskii closure for collisions [e.g., Ricci, J. Plasma Phys. 81, (2015) 435810202]

Examples:

SOLT [Russell et al. Phys. Plasma 16 (2009) 122304]

HESEL [Nielsen et al. PPCF 59 (2017) 025012; Madsen et al. Phys. Plasmas 23 (2016) 032306]

GBS [Halpern et al. J. Comp. Phys. 315 (2016) 388]

Hermes [Dudson et al. PPCF 59 (2017) 054010]

Tokam3X [Tamain et al. PPCF 57 (2015) 0054014]

Examples from HESEL

$\checkmark$ The codes predict plasma impact on material surfaces, including intermittent bursts - They provide:

$\checkmark$ heat source term for the design of the divertor and first wall

$\checkmark$ input for materials modelling 


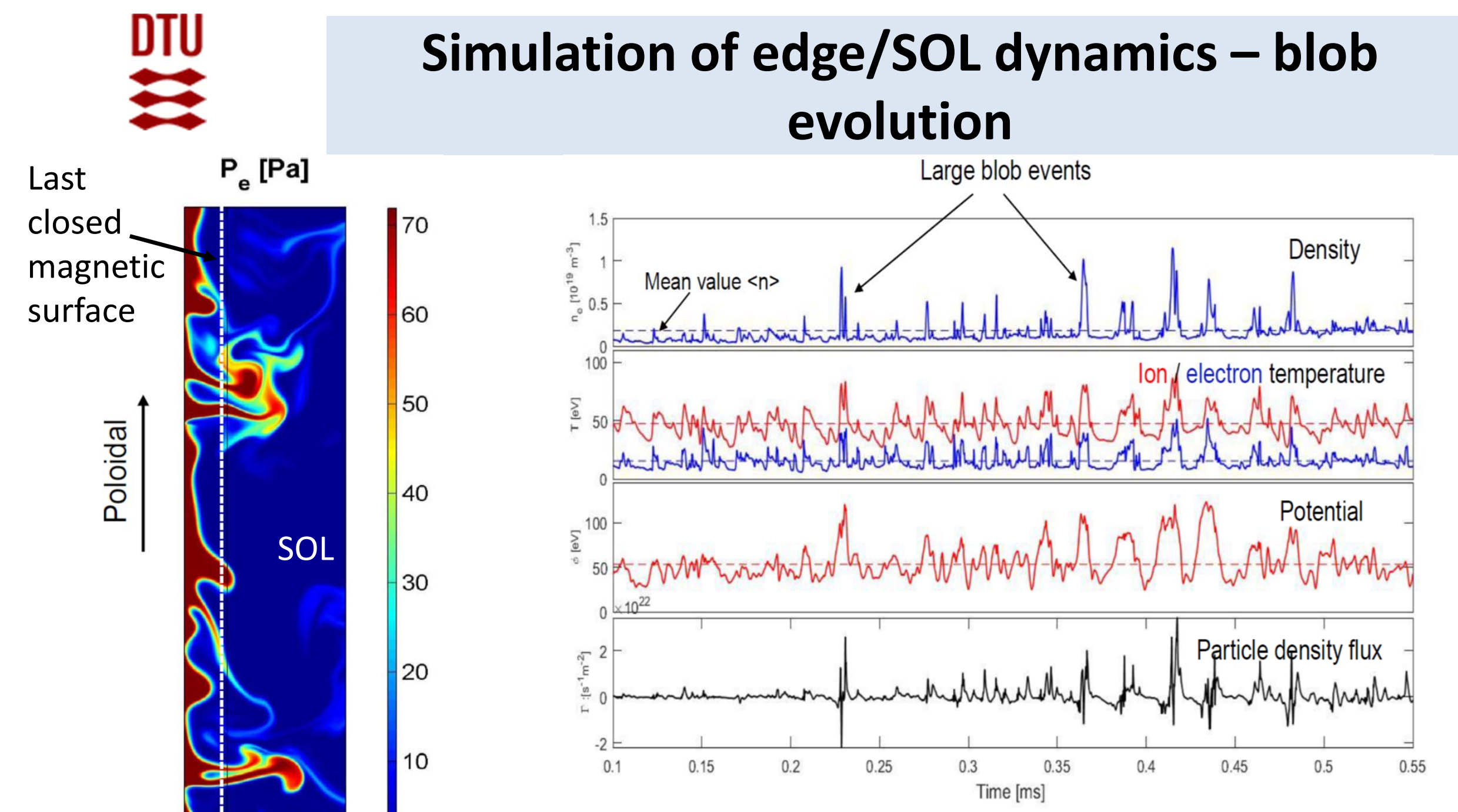

Particle density, electron and ion temperature, plasma potential, and the particle density flux monitored at a "probe" in mid-SOL. Strongly intermittent fluctuations with peak values several times the mean value - make it difficult to predict the maximum heat flux 


\section{Fusion reactor conceptual design}

- During the early stages of reactor design:

- Must explore alternative designs

- Perform important parameter trade-offs

- Optimise sub-system and reactor design and performance

- This is difficult if the design time is of the order of months...

- Very few design iterations

- Difficult to investigate alternatives

\section{Need: Reactor design frameworks}

- Replicate and automate the reactor design process

- Accelerate design point definition by orders of magnitude

- Enable optimisation

- Important because:

- Can accelerate the design cycle and enable actual optimisation of reactor design and sub-systems

- Can connect various layers of knowledge and modelling (e.g. from systems codes to neutronics codes) ensuring consistency between the different models we use to design reactors.
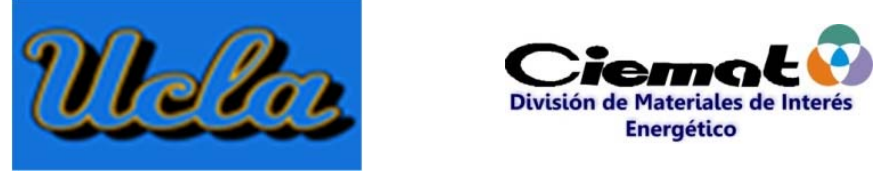


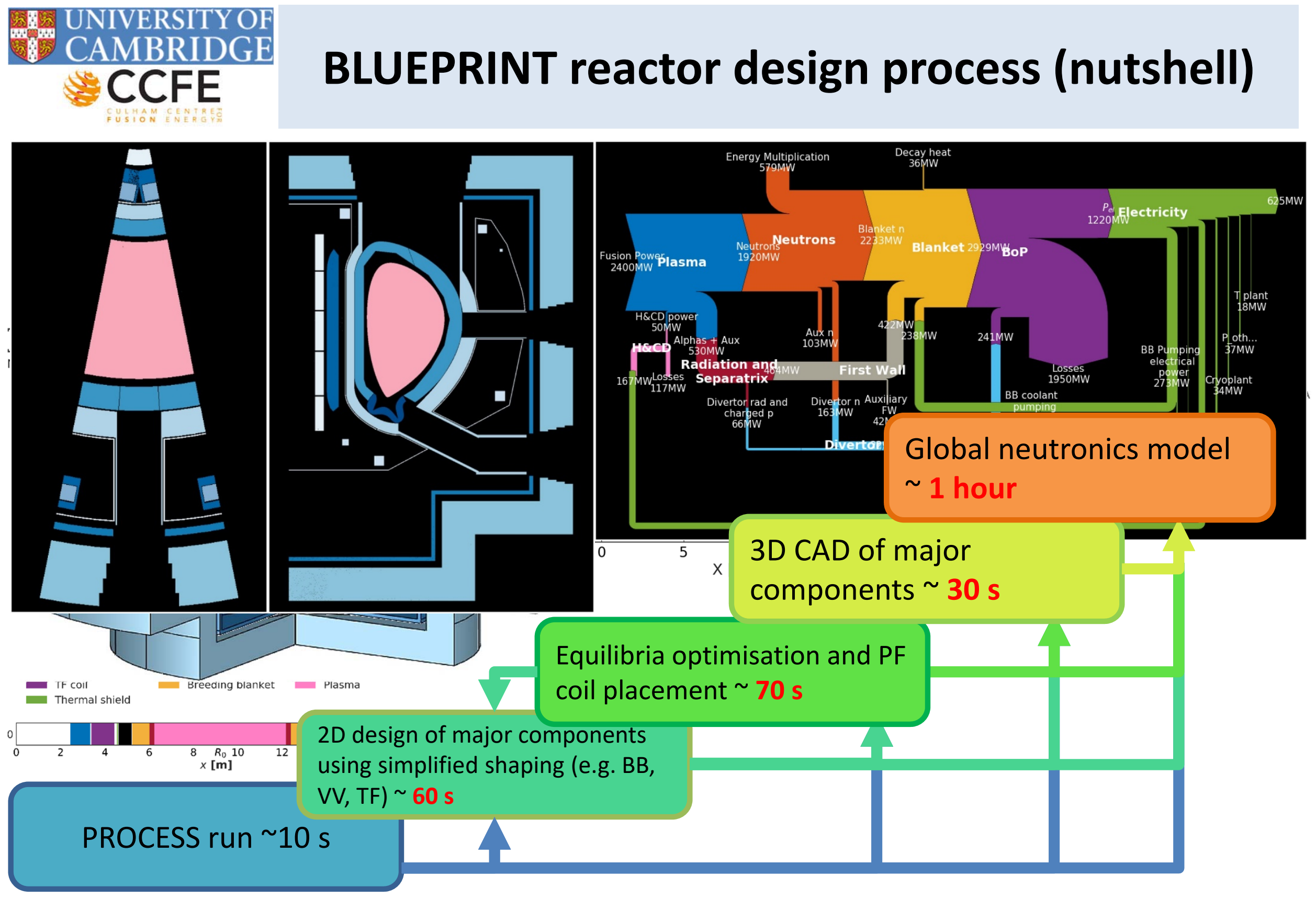

BLUEPRINT: a novel approach to fusion reactor design 


\section{computational tool to fully serve our needs?}

- Despite significant progress in the development of computational tools in each particular area, integration into a single computational tool is premature as no component has reached the desired performance level

- Common requirements for each particular area:

- Multi-physics capability to cover a range of physical processes from single to multi-effect phenomena at different length scales

- Efficiency and accuracy in reproducing the time-dependent behaviour with broad ranges of involved time scales

- $\quad$ Ability to cover ranges up to high values of specific parameters (dimensionless numbers, neutron dose and doserate, stress and strain, temperature gradients ...)

- $\quad$ Ability to handle complex geometries

- $\quad$ Sufficient computational speed

- Complete verification and extensive validation

- This should not stop from aiming towards a strong coupling of codes, even though the first attempts may need to be limited to simple coupling scenarios, or reduced computational models

- Integration strategies and attributes need to be evaluated in dedicated studies; at least in materials machine learning techniques may open new ways

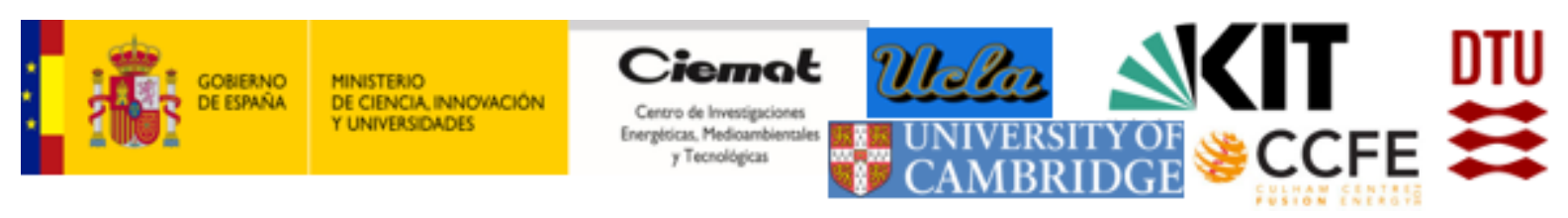




\section{On the role of integrated computer} modelling in fusion technology

\section{THANK YOU FOR YOUR ATTENTION}

Sergey Smolentseva ${ }^{a}$ Gandolfo Alessandro Spagnuolo ${ }^{b}$,

Arkady Serikov ${ }^{b}$, Jens Juul Rasmussenc,

Anders H. Nielsen ${ }^{c}$, Volker Naulin', Jaime Marian ${ }^{d}$, Matti Coleman ${ }^{\mathrm{e}, \mathrm{f}}$ and Lorenzo Malerbag,"

aUCLA, Mechanical \& Aerospace Eng. Dept., 48-121 E4, Los Angeles, CA 90095-1597,USA

${ }^{b} K I T$, Hermann-von-Helmholtz-Platz 1, 76344 Eggenstein-Leopoldshafen, Germany

cPPFE, Dept. Physics, Technical University of Denmark, DK-20800 Kgs. Lyngby, Denmark

dUCLA, Materials Science \& Eng. Dept., 410 Westwood Plaza, Los Angeles, CA 90095-1595, USA

eUKAEA, Culham Science Centre, Abingdon, OX14 3DB, United Kingdom

fUniversity of Cambridge, Trumpington Street, Cambridge, CB2 1PZ, United Kingdom

${ }^{g}$ CIEMAT, Energy Materials Division, Avda. Complutense 40, 28040 Madrid, Spain

lorenzo.malerba@ciemat.es

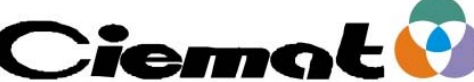

División de Materiales de Interés

Energético 